\title{
Graphene calling
}

\section{Three years after the first experimental results, graphene promises more fascinating physics and dream applications.}

At times when vast amounts of funding are invested in large experimental facilities and centres of excellence, the fact that solid science can emerge out of pencils and adhesive tapes seems surprising. However, three years after it was first isolated by mechanical exfoliation of graphite $^{1}$, there is no doubt that graphene, a single layer of carbon atoms densely packed into a benzene-ring structure, is an excellent playground for condensed-matter physicists and materials scientists.

Actual experimental data are still few and far between, but it has been unequivocally demonstrated that this material exhibits physical properties at the interface between traditional condensed-matter physics and the theory of relativistic electrons in vacuum quantum electrodynamics ${ }^{2,3}$. The interest in this material has grown exponentially, and the number of publications per year doubled in 2006, to a total of almost 350 papers according to ISI. This year the American Physical Society March meeting includes eight sessions on the topic. Nature Materials, in a joint effort with other Nature journals, has dedicated a web-focus to the subject, with the aim of highlighting recent achievements in the field ${ }^{4}$.

In this issue, Andre Geim and Kostya Novoselov outline the brief but intense history of graphene ${ }^{5}$, following their first demonstration of the electric field effect ${ }^{1}$. The authors' review ranges from the obstacles in stabilizing a strictly two-dimensional crystal, through the unusual transport properties that have so far been observed, to the dreams of possible applications to electronic devices. A clear example of the on-going emergence of unusual electronic properties is the interaction between electrons and nuclei ${ }^{6}$, which is yet another validation of the

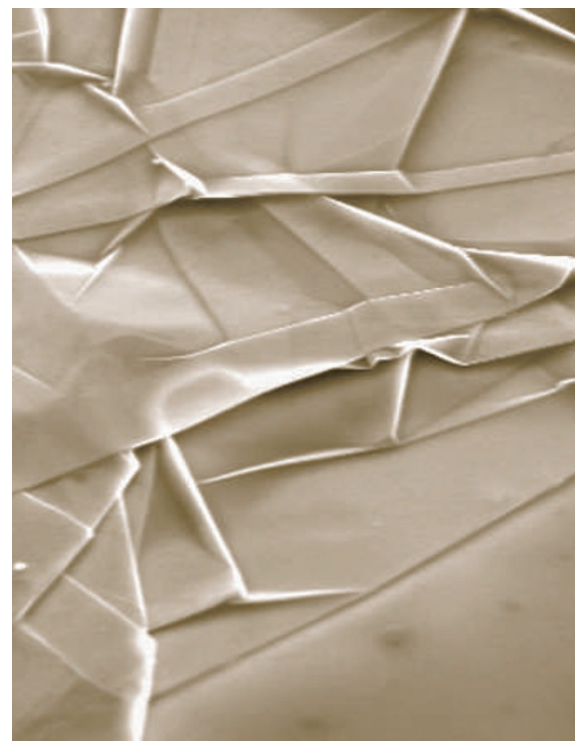

Graphene thrown on a silicon wafer like a veil of silk on a surface. More intriguing science seems to be hiding within the weave of this carbon sheet.

analogy between the physics of graphene and quantum electrodynamics ${ }^{7}$.

Although we can be confident that graphene still has a lot to offer in terms of fundamental physics, the situation is not as clear regarding applications. Undoubtedly there are aspects of this material that make it fascinating for electronics ${ }^{5,8}$. Given the high quality of the carbon lattice, electrons move ballistically, that is, without scattering, for several hundreds of nanometres even at room temperature. This feature makes graphene a unique material for the development of high-frequency transistors up to the terahertz domain. Alternatively, the two-dimensional crystal can be considered as a miniature printed circuit board, from which every element of a nanoscale electronic circuit, like a singleelectron transistor, can be obtained.
However, such devices have not yet been made. Although the possibility of tuning the charge density would in principle allow the implementation of standard semiconductor electronics, graphene is always metallic. So, unless a convenient way of making it semiconducting is demonstrated, its use for devices remains debatable. Another major obstacle is the need for a reliable method to produce graphene sheets in large amounts. The original method based on exfoliation works quite well for fundamental studies, but is not suitable for large-scale applications. Alternative routes have already been proposed. One of these is based on the heat treatment of silicon carbide wafers that produces carbon films, one layer at a time ${ }^{9}$. It has also been shown that immersing graphite oxide in water results in the dispersion of graphene sheets that can then be stabilized by amphiphilic polymers and incorporated into nanocomposites ${ }^{10}$. Both strategies have shown promising results, but high-quality crystals have not been demonstrated so far.

As is clear from the programme of the APS meeting, work on graphene is at present mostly about fundamental research. But the interest is also growing for potential applications. At Nature Materials, we are looking forward to following the developments of research on both aspects of this unusual and intriguing material.

\section{References}

1. Novoselov, K. S. et al. Science 306, 666-669 (2004).

2. Novoselov, K. S. et al. Nature 438, 197-200 (2005).

3. Zhang, Y., Tan, J. W., Stormer, H. L. \& Kim, P. Nature 438, 201-204 (2005).

4. http://www.nature.com/conferences/aps

5. Geim, A. K. \& Novoselov, K. S. Nature Mater. 6, 183-191 (2006).

6. Pisana, S. et al. Nature Mater. 6, 198-201 (2007).

7. Castro Neto, A. Nature Mater. 6, 176-177 (2007).

8. Ball. P. Nature Mater. 5, 434 (2006).

9. Berger, C. et al. Science 312, 1191-1196 (2006).

10. Stankovich, S. et al. Nature 442, 282-286 (2006). 